\title{
Mudanças no atendimento de enfermagem aos idosos em Instituições de Longa Permanecia para Idosos durante a pandemia do covid-19
}

\author{
Changes in nursing care for the elderly in long-term care institutions during the covid-19 pandemic \\ Cambios en la atención de enfermería para los ancianos en instituciones de cuidado a largo plazo \\ durante la pandemia covid-19
}

Recebido: 10/07/2021 | Revisado: 16/07/2021 | Aceito: 16/07/2021 | Publicado: 25/07/2021

\author{
Natasha Vila Chã \\ ORCID: https://orcid.org/0000-0002-9781-454X \\ Associação Brasileira de Enfermeiros Cientistas, Brasil \\ E-mail: natashavilacha@ hotmail.com \\ Priscila Gramata da Silva Vitorino \\ ORCID: https://orcid.org/0000-0002-1201-6945 \\ Associação Brasileira de Enfermeiros Cientistas, Brasil \\ E-mail: prigramaenf@yahoo.com.br \\ Judith Victoria Castillo Mejía \\ ORCID: https://orcid.org/0000-0001-5257-6878 \\ Associação Brasileira de Enfermeiros Cientistas, Brasil \\ E-mail: iker07castillo@live.com \\ Victor Hugo de Paula Flauzino \\ ORCID: https://orcid.org/0000-0001-5156-0030 \\ Associação Brasileira de Enfermeiros Cientistas, Brasil \\ E-mail: prof.victorflauzino@unyleya.edu.br \\ Thays Vieira Cusato \\ ORCID: https://orcid.org/0000-0002-3766-6530 \\ Associação Brasileira de Enfermeiros Cientistas, Brasil \\ E-mail: thays.vieira.cusato@gmail.com \\ Daiana Moreira Gomes \\ ORCID: https://orcid.org/0000-0001-9387-0619 \\ Associação Brasileira de Enfermeiros Cientistas, Brasil \\ E-mail: daigomes_87@ hotmail.com \\ Daniele Vignoli Ribeiro \\ ORCID: https://orcid.org/0000-0001-5296-8302 \\ Associação Brasileira de Enfermeiros Cientistas, Brasil \\ E-mail: vignoliribeiro@gmail.com \\ Luana de Oliveira Hernandes \\ ORCID: http://orcid.org/0000-0001-8828-7146 \\ Associação Brasileira de Enfermeiros Cientistas, Brasil \\ E-mail: luanaoliveira2306@gmail.com \\ Thais Oliveira de Paula Lima \\ ORCID: https://orcid.org/0000-0002-0706-1098 \\ Associação Brasileira de Enfermeiros Cientistas, Brasil \\ E-mail: thaisoplima@gmail.com \\ Jonas Magno dos Santos Cesário \\ ORCID: https://orcid.org/0000-0003-1785-3555 \\ Associação Brasileira de Enfermeiros Cientistas, Brasil \\ E-mail: prof.jonasmagno@unyleya.edu.br
}

\begin{abstract}
Resumo
As Instituições de Longa Permanecia para Idosos (ILPI) oferecem a atenção integral e suporte para indivíduos que necessitem de cuidados especializados e que não podem ser assistidos pela própria família. Porém, durante a pandemia do covid-19, estes ambientes demonstraram ser "alvos fáceis" para o novo coronavírus e por isso, exigiu de toda a equipe de saúde, uma resposta e adaptação rápidas. O principal objetivo deste estudo foi identificar as mudanças no atendimento de enfermagem aos idosos em ILPI durante a pandemia do coronavírus. Trata-se de uma pesquisa descritiva, com busca de artigos realizada em bibliotecas digitais. Os estudos analisados indicaram que os pacientes apresentaram quadro clínicos diferenciados, como aparecimentos de sintomas físicos e mentais, em menor ou maior gravidade. O enfermeiro como membro principal do sistema de gerenciamento das ILPI, contribui para coordenar a implantação de medidas de cuidado, com o propósito de promover a saúde, prevenir e controlar a disseminação do vírus. Cabe ao enfermeiro adotar estratégias para diminuir o risco de infecção e mortalidade. É evidente os enormes desafios encontrados por tais profissionais em implantar medidas de prevenção e cuidado contra
\end{abstract}


uma doença pouco conhecida naquele momento e em tão pouco tempo. Foi possível constatar que, quanto maior a capacitação e organização da assistência do pessoal de enfermagem melhores são os resultados obtidos na segurança da assistência a população idosa e que o enfermeiro precisa estar preparado para agir em diferentes situações, até mesmo em emergências.

Palavras-chave: Geriatria; Fatores de risco; Covid-19.

\begin{abstract}
Long Stay Institutions for the Elderly (LTCI) offer comprehensive care and support for individuals who need specialized care and cannot be assisted by their own family. However, during the covid-19 pandemic, these environments proved to be "easy targets" for the new coronavirus and, therefore, required a rapid response and adaptation from the entire healthcare team. The main objective of this study was to identify changes in nursing care for the elderly in LSIE during the coronavirus pandemic. This is a descriptive research, with a search for articles carried out in digital libraries. The studies analyzed indicated that the patients presented different clinical conditions, such as the appearance of physical and mental symptoms, to a lesser or greater degree. The nurse, as the main member of the LSIE management system, helps to coordinate the implementation of care measures, with the purpose of promoting health, preventing, and controlling the spread of the virus. It is up to the nurse to adopt strategies to reduce the risk of infection and mortality. The enormous challenges faced by these professionals in implementing prevention and care measures against a little-known disease at that time and in such a short time are evident. It was found that the greater the training and organization of nursing staff care, the better the results obtained in the safety of care for the elderly population and that nurses need to be prepared to act in different situations, even in emergencies.
\end{abstract}

Keywords: Geriatrics; Risk factors; Covid-19.

\title{
Resumen
}

Las Instituciones de Larga Estancia para Ancianos (LTCI, por sus siglas en inglés) ofrecen atención y apoyo integrales para las personas que necesitan atención especializada y no pueden ser asistidas por su propia familia. Sin embargo, durante la pandemia de covid-19, estos entornos demostraron ser "blancos fáciles" para el nuevo coronavirus y, por lo tanto, requirieron una rápida respuesta y adaptación de todo el equipo de atención médica. El objetivo principal de este estudio fue identificar cambios en la atención de enfermería a los ancianos en LSIE durante la pandemia de coronavirus. Se trata de una investigación descriptiva, con búsqueda de artículos realizada en bibliotecas digitales. Los estudios analizados indicaron que los pacientes presentaban diferentes condiciones clínicas, como la aparición de síntomas físicos y mentales, en menor o mayor grado. La enfermera, como miembro principal del sistema de gestión de la LSIE, contribuye a coordinar la implementación de las medidas de atención, con el propósito de promover la salud, prevenir y controlar la propagación del virus. Corresponde a la enfermera adoptar estrategias para reducir el riesgo de infección y mortalidad. Son evidentes los enormes retos a los que se enfrentan estos profesionales para implementar medidas de prevención y atención frente a una enfermedad poco conocida en ese momento y en tan poco tiempo. Se pudo observar que cuanto mayor es la capacitación y organización de la atención brindada por el personal de enfermería, mejores son los resultados obtenidos en la seguridad del cuidado de la población anciana y que las enfermeras deben estar preparadas para actuar en diferentes situaciones, incluso en emergencias.

Palabras clave: Geriatría; Factores de riesgo; Covid-19.

\section{Introdução}

A população idosa cresce gradativamente no Brasil e um dos fatores que contribui para esse crescimento é o aumento na expectativa de vida da população, relacionada a melhores condições e acesso aos serviços de saúde. Durante o processo de envelhecimento, o ser humano tenta se readaptar a um novo modelo de vida, pois há uma diminuição na sua capacidade de adaptação e na sua capacidade motora. Por esse motivo, é de suma importância acompanhar o desenvolvimento desse individuo, para garantir que ele percorra esse processo de forma saudável, e que, sua independência e capacidade de realizar o autocuidado sejam estimulados (Santos, Ribeiro \& Lopes, 2020).

O convívio com o idoso pode ser algo bastante desafiador, pois, é necessário o desenvolvimento de diferentes hábitos de cuidado, somado a muita dedicação e paciência. Geralmente, a família é a responsável por acompanhar e dar todo o suporte necessário para o bem-estar do idoso. Porém, a falta de habilidade em relação a algum tipo de cuidado específico, assim como a dificuldade de compreensão do comportamento do idoso, ou até mesmo, a falta de condição financeira, faz com que muitas famílias não aceitem fazer o papel de cuidadores e tentam buscar ajudar. Para respaldar e ajudar os idosos com as atividades do dia a dia, as Instituições de Longa Permanecia para Idosos (ILPI) oferecem a atenção integral e suporte para esses indivíduos. 
As ILPI se tornaram os "principais alvos" do covid-19 durante a pandemia, pois devido a fragilidade destes pacientes, tais locais demonstraram ser o ambiente ideal para que o vírus se espalhasse e levasse inúmeros idosos a óbito. Os idosos têm uma maior possibilidade de desenvolver complicações, principalmente, quando já apresentam doenças de base como insuficiência cardíaca, hipertensão e diabetes. Essas comorbidades, ao afetar essa faixa etária, fazem com que os idosos fiquem ainda mais vulneráveis e expostos a qualquer tipo de infecção. Devido a esse histórico, observou-se que houve um aumento na taxa de transmissibilidade superior a $60 \%$ com mortalidade associada a infecção pelo coronavírus, em indivíduos com mais de 60 anos (Moraes et al., 2020).

A doença denominada covid-19 foi originada pela infecção do coronavírus SARSCoV-2 (Severe Acute Respiratory Syndrome Coronavirus 2), identificado primeiramente na cidade de Wuhan, na China. É uma infecção que atua de forma rápida e que acomete o sistema respiratório, no qual apresenta sintomas semelhantes aos da gripe. Contudo, foi possível observar que os pacientes infectados manifestaram desde um quadro gripal, até uma forma avançada da doença, no qual o sistema respiratório é agressivamente atingido. É uma doença potencialmente contagiosa, que é transmitida de indivíduo para indivíduo por meio de contato direto de pessoas sintomáticas e assintomáticas (Oliveira, Machado \& Dadalto, 2020).

A infecção causada pelo coronavírus apresentou-se como um verdadeiro desafio mundial, por se tratar de uma doença respiratória com alta capacidade de transmissão e que apresentou letalidade superior na população idosa. As ILPI tiveram que criar e efetivar medidas preventivas para controlar a incidência de infecção e evitar ou reduzir ao máximo que os idosos, cuidadores e profissionais que viviam e frequentavam esses estabelecimentos, fossem infectados pelo vírus, com o objetivo de reduzir a morbimortalidade entre os idosos nessas unidades (Menezes et al., 2020).

O combate ao covid-19 nas ILPI exigiu grande esforço dos profissionais de saúde, principalmente da equipe de enfermagem. A velocidade de propagação dessa nova doença impôs um ritmo de trabalho para o qual a maioria das instituições de saúde não estavam preparadas, o que alterou de maneira significativa a rotina assistencial dos enfermeiros. Devido ao grande impacto que a covid-19 causou, foi de extrema importância que protocolos e/ou orientações fossem adotados nas ILPI, assim como a promoção de medidas de controle durante o cuidado aos idosos residentes nessas instituições. Além disso, foi necessário controlar o fluxo de profissionais que frequentavam as ILPI e diferentes ambientes, como hospitais e outras unidades de saúde ao mesmo tempo, com o objetivo de diminuir a contaminação cruzada e consequentemente o risco de infecção (Moraes et al., 2020).

Nesse contexto, esse estudo busca responder a seguinte pergunta: qual o impacto da covid-19 no atendimento de enfermagem para idosos em ILPI? Traçou-se então como objetivo, identificar as mudanças no atendimento de enfermagem aos idosos em ILPI durante a pandemia do coronavírus.

\section{Metodologia}

Este estudo é uma pesquisa descritiva, que de acordo com Cesário, Flauzino e Mejia (2020) é direcionada para responder alguma dúvida ou questionamento, que pode incluir artigos científicos publicados em periódicos acadêmicos. Dessa forma, inicialmente buscou-se reunir evidências para responder ao questionamento de pesquisa. Na etapa inicial, a busca foi realizada em bibliotecas digitais, que permitem uma busca em bases de dados próprias por meio da utilização de descritores padronizados. No DeCS (Descritores em Ciências da Saúde), foram encontrados os seguintes descritores: geriatria, fatores de risco e COVID-19.

Os bancos de dados utilizados foram o Google Acadêmico, BVS (biblioteca virtual em saúde) e SciELO (Scientific Electronic Library Online). No Google Acadêmico utilizou-se cada um dos descritores entre aspas (“"). Na BVS foi utilizado a opção pesquisa avançada, selecionada as bases da BDENF (Banco de Dados em Enfermagem), LILACS (Literatura Latino- 
Americana e do Caribe em Ciências da Saúde) e o operador lógico booleano "OR" e "AND". Na SciELO foi utilizada a opção pesquisa avançada e o operador lógico booleano "OR" e "AND".

Foram estabelecidos como critérios de inclusão: artigos acadêmicos publicados entre 2019 e 2021, na língua portuguesa, disponíveis de forma gratuita e nos bancos de dados já mencionados, que respondessem à pergunta de pesquisa. Excluíram-se artigos repetidos encontrados nas bases de dados, resumos, artigos inferiores a 2019 e artigos que não respondiam o problema da pesquisa. A coleta dos dados foi realizada no mês de março, por dois pesquisadores de forma independente. Os resultados das buscas pelos dados e do número final de publicações que irão compor a revisão serão apresentados conforme orientações do Prisma na forma de fluxograma, conforme a Figura 1 a seguir (Peters, 2015).

Figura 1. Fluxograma de prisma.

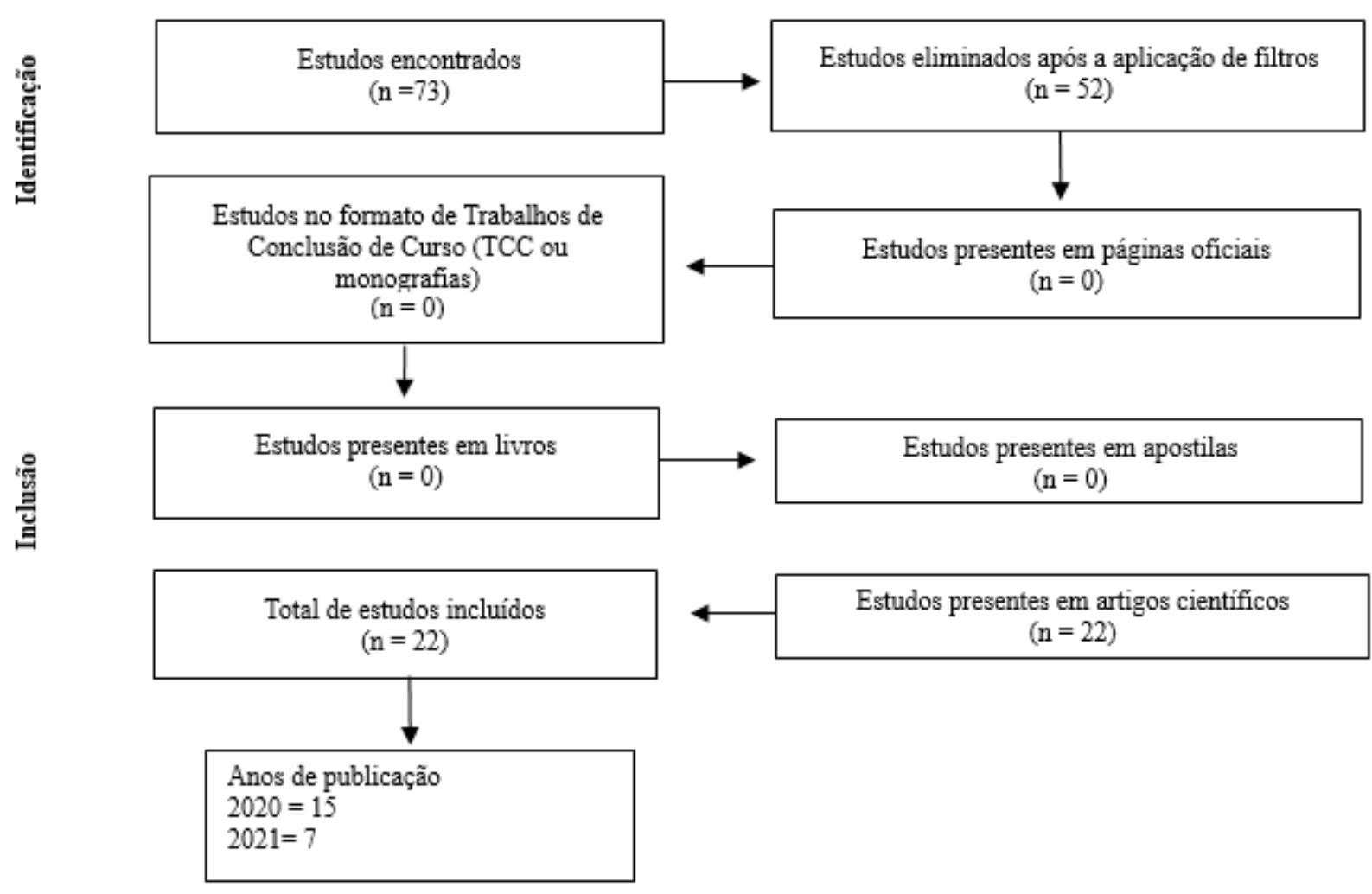

Fonte: Autores (2021).

Inicialmente foram encontrados 73 estudos e após a aplicação dos filtros, conforme os critérios de inclusão e exclusão, foram eliminados 52 estudos. Não foram considerados estudos em páginas oficiais da internet, monografias, apostilas e livros. Ao final foram incluídos 22 artigos científicos.

\section{Resultados}

Para a elaboração da pesquisa foram incluídos 22 artigos, que estão dispostos em periódicos diferentes, nas bases dados do Google Acadêmico, BVS e SciELO. A tabela mostra a quantidade de estudos que foram encontrados durante a pesquisa e estão agrupados por periódicos em suas respectivas quantidades. 
Tabela 1. Distribuição de artigos de acordo com o periódico de publicação.

\begin{tabular}{ll|}
\hline Periódicos & $\mathbf{N}^{\circ}$ \\
\hline Revista, Society and Development & 3 \\
Revista Nursing & 3 \\
Disciplinarum Scientia & 1 \\
Revista Diálogos em Saúde & 1 \\
Revista Kairós-Gerontologia & 1 \\
Revista Brasileira de Geriatria e Gerontologia. & 1 \\
Revista Eletrônica Acervo Saúde & 1 \\
Revista de Enfermagem do Centro Oeste Mineiro & 1 \\
Congitare Enfermagem & 1 \\
Escola Anna Nery & 1 \\
Revista. Enfermagem UFPE & 1 \\
Revista de Divulgação Científica Sena Aires & 1 \\
Revista Pensar Acadêmico UNIFACIG & 1 \\
Revista Rede de Enfermagem do Nordeste (Rene) & 1 \\
Revista Interface & 1 \\
Editora Realize & 1 \\
Jiência e Saúde Coletiva & 1 \\
\hline Total & 1 \\
\hline
\end{tabular}

Fonte: Autores (2021).

Com a finalidade de apresentar os resultados encontrados durante o desenvolvimento da pesquisa, foi elaborado o Quadro 1, que contêm os artigos incluídos nesse estudo, e, estão separados pelas variáveis: autor, título, objetivo central e tipo de estudo. Os estudos foram organizados de forma que contribuíssem na comparação e análise dos conteúdos selecionados, assim como, colaborar para a interpretação dos resultados.

Quadro 1. Artigos inclusos neste estudo.

\begin{tabular}{|c|c|c|c|}
\hline Autor/ano & Título & Objetivos & Tipo de estudo \\
\hline Goulart et al., 2020 & $\begin{array}{l}\text { A pandemia por covid-19 e o } \\
\text { paciente idoso: quais as } \\
\text { necessidades de atenção em saúde } \\
\text { para esse grupo populacional? }\end{array}$ & $\begin{array}{l}\text { Abordar as diferentes facetas da } \\
\text { vulnerabilidade do paciente idoso } \\
\text { durante a pandemia por covid-19, com } \\
\text { foco nos impactos biológicos, } \\
\text { psicológicos e sociais da doença. }\end{array}$ & Pesquisa bibliográfica. \\
\hline Melo et al., 2020 & $\begin{array}{l}\text { Ações educativas aos idosos frente } \\
\text { a pandemia de sars-cov-2: estudo } \\
\text { de revisão. }\end{array}$ & $\begin{array}{l}\text { Realizar uma revisão de literatura sobre } \\
\text { ações educativas aos idosos frente a } \\
\text { pandemia de SARS-CoV- } 2 \text {. }\end{array}$ & Revisão de literatura. \\
\hline Santos, et al 2020 & $\begin{array}{l}\text { Análise da estrutura assistencial em } \\
\text { saúde para com os idosos na } \\
\text { vigência da covid-19. }\end{array}$ & $\begin{array}{l}\text { Reunir o espectro informacional de base } \\
\text { científica com uma visão direcionada } \\
\text { em relação à análise estrutural do } \\
\text { âmbito de saúde no sentido do cuidado } \\
\text { para com os idosos em períodos de } \\
\text { pandemia, em específico em meio ao }\end{array}$ & $\begin{array}{lll}\text { Revisão } & \text { integrativa } & \text { da } \\
\text { literatura. } & & \\
\end{array}$ \\
\hline
\end{tabular}




\begin{tabular}{|c|c|c|c|}
\hline & & surto de covid-19. & \\
\hline Leite et al., 2020 & $\begin{array}{l}\text { Cuidados nutricionais para idosos } \\
\text { com doença pulmonar obstrutiva } \\
\text { crônica em tempos de covid- } 19 \text {. }\end{array}$ & $\begin{array}{l}\text { Descrever o estado e as necessidades } \\
\text { nutricionais relacionados ao processo de } \\
\text { envelhecimento e da doença pulmonar } \\
\text { obstrutiva crônica (DPOC) na população } \\
\text { idosa em tempos de covid-19. }\end{array}$ & $\begin{array}{l}\text { Pesquisa bibliográfica } \\
\text { descritiva e narrativa. }\end{array}$ \\
\hline Cardoso et al., 2020 & 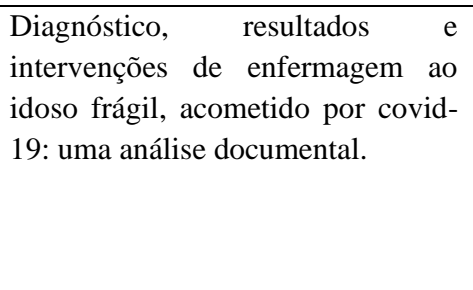 & $\begin{array}{l}\text { Elencar os principais diagnósticos, } \\
\text { resultados } \mathrm{e} \text { intervenções de } \\
\text { enfermagem ao idoso frágil em } \\
\text { Instituição de Longa Permanência } \\
\text { (ILPI) e aos idosos em domicílio } \\
\text { acometidos pelo novo coronavírus } \\
\text { (covid-19). }\end{array}$ & $\begin{array}{lr}\text { Revisão } & \text { documental, } \\
\text { abordagem } & \text { com } \\
\text { cunhlitativa, de } & \text { de } \\
\text { cunho desitivo e exploratório. }\end{array}$ \\
\hline Lucchini et al., 2020 & $\begin{array}{l}\text { Fatores de risco para o suicídio em } \\
\text { idosos antes e durante o período de } \\
\text { confinamento por covid- } 19 \text {. }\end{array}$ & $\begin{array}{l}\text { Analisar os fatores relacionados à saúde } \\
\text { mental e de risco para suicídio entre } \\
\text { idosos e verificar sua associação com a } \\
\text { pandemia de covid- } 19 \text {. }\end{array}$ & Revisão bibliográfica. \\
\hline Barbosa et al., 2020 & $\begin{array}{l}\text { Incidência e mortalidade por covid- } \\
19 \text { na população idosa brasileira e } \\
\text { sua relação com indicadores } \\
\text { contextuais: um estudo ecológico. }\end{array}$ & $\begin{array}{l}\text { Analisar a incidência e mortalidade por } \\
\text { covid-19 na população idosa no Brasil e } \\
\text { sua relação com variáveis contextuais. }\end{array}$ & $\begin{array}{l}\text { Estudo observacional, ecológico } \\
\text { e analítico. }\end{array}$ \\
\hline Gomes et al., 2020 & $\begin{array}{l}\text { Qualidade de vida de idosos antes e } \\
\text { durante a pandemia da covid-19 e } \\
\text { expectativa na pós-pandemia. }\end{array}$ & $\begin{array}{l}\text { Conhecer as opiniões de um grupo de } 67 \\
\text { idosos que, anteriormente à pandemia da } \\
\text { covid-19, foram atendidos em } \\
\text { ambulatórios de geriatria de } \\
\text { universidade, interrogando-se quanto } \\
\text { aos sentimentos surgidos com a } \\
\text { pandemia e à qualidade de vida (QV) } \\
\text { em três momentos (antes e durante a } \\
\text { pandemia e a expectativa de como será } \\
\text { na pós-pandemia). }\end{array}$ & $\begin{array}{l}\text { Estudo exploratório descritivo, } \\
\text { transversal, com abordagem } \\
\text { quanti-qualitativa. }\end{array}$ \\
\hline Monteiro et al., 2020 & $\begin{array}{l}\text { Recomendações aos cuidadores e } \\
\text { familiares de idosos mediante a } \\
\text { covid-19. }\end{array}$ & 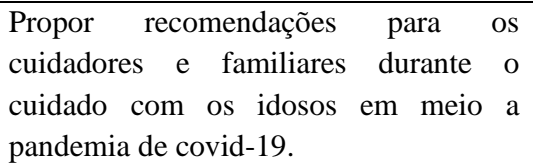 & $\begin{array}{l}\text { Revisão narrativa com análise } \\
\text { reflexiva da literatura vigente. }\end{array}$ \\
\hline Souza et al., 2021 & $\begin{array}{l}\text { Vulnerabilidade e fatores de risco } \\
\text { associados para covid-19 em idosos } \\
\text { institucionalizados. }\end{array}$ & $\begin{array}{l}\text { Identificar a associação de fatores de } \\
\text { risco para covid-19 com a } \\
\text { vulnerabilidade em pessoas idosas } \\
\text { residentes em ILPI. }\end{array}$ & $\begin{array}{l}\text { Estudo descritivo, exploratório, } \\
\text { com abordagem quantitativa. }\end{array}$ \\
\hline Lima et al., 2021 & $\begin{array}{l}\text { Processo de enfermagem para } \\
\text { pacientes com manifestações } \\
\text { respiratórias da covid-19 }\end{array}$ & $\begin{array}{l}\text { Discutir sobre o } \quad \text { Processo de } \\
\text { Enfermagem diante dos aspectos } \\
\text { clínicos respiratórios da covid-19 }\end{array}$ & Estudo qualitativo, descritivo. \\
\hline Marins et al., 2020 & $\begin{array}{l}\text { A saúde da pessoa idosa no } \\
\text { contexto da pandemia pelo } \\
\text { coronavírus: considerações para a } \\
\text { enfermagem }\end{array}$ & $\begin{array}{l}\text { Refletir sobre a saúde da pessoa idosa } \\
\text { na pandemia pela covid-19, para a } \\
\text { elaboração de orientações de } \\
\text { enfermagem dirigidas a Atenção } \\
\text { Primária à Saúde e à Assistência } \\
\text { Hospitalar Especializada }\end{array}$ & Estudo de reflexão \\
\hline Reis et al., 2020 & $\begin{array}{l}\text { Atuação da enfermagem no cenário } \\
\text { da pandemia covid-19 }\end{array}$ & $\begin{array}{l}\text { Relatar as experiências, receios e } \\
\text { anseios dos profissionais de } \\
\text { enfermagem que atuam na linha de } \\
\text { frente aos cuidados de pacientes } \\
\text { suspeitos e confirmados do covid-19 }\end{array}$ & $\begin{array}{l}\text { Estudo descritivo, exploratório, } \\
\text { com abordagem qualitativa, do } \\
\text { tipo relato de experiência }\end{array}$ \\
\hline Seixas et al., 2020 & $\begin{array}{l}\text { A crise como potência: os cuidados } \\
\text { de proximidade e a epidemia pela } \\
\text { covid-19 }\end{array}$ & $\begin{array}{l}\text { Tensionar os equívocos do modelo } \\
\text { biomédico hospitalocêntrico privatizante } \\
\text { com base na resposta à epidemia pela } \\
\text { covid-19, que tem como centralidade o } \\
\text { cuidado no hospital e as tecnologias }\end{array}$ & Revisão integrativa de literatura \\
\hline
\end{tabular}




\begin{tabular}{|c|c|c|c|}
\hline & & duras e leve-duras. & \\
\hline Souza et al., 2021 & $\begin{array}{l}\text { Influência da cobertura da atenção } \\
\text { básica no enfrentamento da covid- } \\
19 .\end{array}$ & $\begin{array}{l}\text { Avaliar a influência da cobertura da } \\
\text { atenção básica nos municípios de Santa } \\
\text { Catarina e seus efeitos no enfrentamento } \\
\text { da covid- } 19 \text {. }\end{array}$ & $\begin{array}{l}\text { Estudo observacional } \\
\text { ecológico. }\end{array}$ \\
\hline Borges et al., 2021 & $\begin{array}{lcr}\text { Percepções e vivências } & \text { de } \\
\text { enfermeiros } & \text { sobre o } \quad \text { seu } \\
\text { desempenho na pandemia da covid- } \\
19 .\end{array}$ & $\begin{array}{l}\text { Descrever a percepção e vivências dos } \\
\text { enfermeiros sobre o seu desempenho } \\
\text { durante a pandemia da covid- } 19 \text {. }\end{array}$ & Estudo qualitativo. \\
\hline $\begin{array}{l}\text { Alexandre, Nunes } \\
2020\end{array}$ & $\begin{array}{l}\text { Problematização sobre a pandemia } \\
\text { da covid-19 como auxílio na } \\
\text { formação de enfermeiras/os. }\end{array}$ & $\begin{array}{l}\text { Desenvolver estratégias para inserção do } \\
\text { conteúdo covid-19 nas disciplinas que } \\
\text { compõe a matriz do Curso de } \\
\text { Enfermagem do Centro Universitário } \\
\text { São Camilo. }\end{array}$ & $\begin{array}{l}\text { Pesquisa qualiquantitativa, com } \\
\text { revisão bibliográfica e pesquisa } \\
\text { de campo }\end{array}$ \\
\hline Santos et al., 2020 & $\begin{array}{l}\text { Repercussões da covid-19 para o } \\
\text { cotidiano da pessoa idosa. }\end{array}$ & $\begin{array}{l}\text { Refletir sobre as repercussões da covid- } \\
19 \text { para a vida da pessoa idosa. }\end{array}$ & Estudo de abordagem reflexiva. \\
\hline $\begin{array}{l}\text { Hammerschmidt \& } \\
\text { Santana. } 2020\end{array}$ & $\begin{array}{l}\text { Saúde do idoso em tempos de } \\
\text { pandemia covid-19. }\end{array}$ & $\begin{array}{l}\text { Abordar de forma reflexiva e crítica } \\
\text { aspectos relacionados à saúde do idoso } \\
\text { nos tempos de pandemia covid- } 19 \text {. }\end{array}$ & Revisão bibliográfica \\
\hline $\begin{array}{ll}\text { Bitencourt } & \& \\
\text { Andrade, } 2021 & \end{array}$ & $\begin{array}{l}\text { Trabalhadoras da saúde face à } \\
\text { pandemia: por uma análise } \\
\text { sociológica do trabalho de cuidado. }\end{array}$ & $\begin{array}{l}\text { Discutir o cuidado de trabalhadoras da } \\
\text { área da saúde em face da covid- } 19 \text {. }\end{array}$ & Revisão integrativa de literatura \\
\hline $\begin{array}{l}\text { Rodríguez, et al., } \\
2021\end{array}$ & $\begin{array}{l}\text { Vacinação contra influenza no } \\
\text { enfrentamento da covid-19: } \\
\text { integração ensino-serviço para } \\
\text { formação em enfermagem e } \\
\text { saúde. }\end{array}$ & 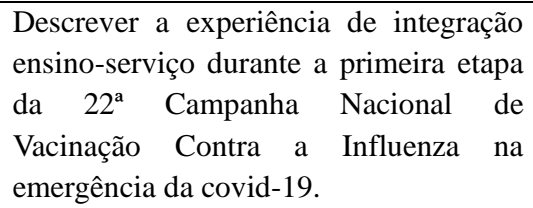 & Relato de experiência. \\
\hline Souza et al., 2021 & $\begin{array}{l}\text { Influência da cobertura da atenção } \\
\text { básica no enfrentamento da covid- } \\
19 .\end{array}$ & $\begin{array}{l}\text { Avaliar a influência da cobertura da } \\
\text { atenção básica nos municípios de Santa } \\
\text { Catarina e seus efeitos no enfrentamento } \\
\text { da covid- } 19 \text {. }\end{array}$ & $\begin{array}{l}\text { Estudo observacional } \\
\text { ecológico. }\end{array}$ \\
\hline
\end{tabular}

Fonte: Autores (2021).

\section{Discussão}

As ILPI oferecem uma assistência integral à população idosa que é totalmente dependente de cuidados. Porém, durante a pandemia do covid-19, observou-se que esses ambientes apresentam um maior risco para os idosos que estão internados, pois o alto fluxo de pessoas que trabalham nessas instituições, assim como a falta de treinamento da equipe multiprofissional, é capaz de proliferar o vírus para uma população vulnerável, frágil, dependente e que, em sua maioria possuem doenças crônicas (Monteiro, et al., 2020).

Os casos de contaminação por covid-19 apresentaram-se de diferentes formas, desde casos assintomáticos, aqueles com sinais e sintomas leves, até a forma mais grave da infecção, que exigem internação e suporte respiratório. O acompanhamento dos pacientes acometidos pelo covid-19, contribuiu para a identificação precoce dos sinais de agravamento da doença. Isso possibilitou a elaboração de estratégias de cuidados mais adequadas. As medidas preventivas foram implantadas com o objetivo de controlar a disseminação do vírus entre a população (Souza et al., 2021).

Em ambientes como os das ILPI, as chances de contaminação foram ainda maiores, e, com o objetivo de evitar ainda mais o aumento de casos de idosos infectados pelo covid-19, a Agência Nacional de Vigilância Sanitária (ANVISA), lançou uma nota técnica para orientar como prevenir e controlar a nova infecção causada pelo coronavírus. Por essa razão, medidas de prevenção e controle foram adotadas por essas instituições, durante o cuidado aos residentes e o foco foi controlar casos de idosos suspeitos, que apresentavam os sintomas da doença, como também, casos de idosos diagnosticados pelo vírus (Santana et al., 2020). 
A pandemia exigiu diferentes atribuições e habilidades dos profissionais de saúde, para lidar com a complexidade e incerteza ocasionada pela contaminação do vírus. A enfermagem, por fazer parte do sistema de gestão de cuidado nas ILPI assumiram a linha de frente e vivenciaram experiências desafiadoras. $\mathrm{O}$ enfermeiro, por ser um dos principais responsáveis em participar de todo o processo de cuidado do paciente, precisou reestruturar suas rotinas assistenciais e desenvolver planejamento estratégicos para enfrentar a doença. Porém, diante de imenso trabalho, muito profissionais vivenciaram problemas como esgotamento emocional, número reduzido de profissionais, carga horária excessiva de trabalho, mudança na forma de atuação da equipe e escassez de recursos materiais. Além disso, os profissionais tiveram que lidar diretamente com a saúde mental dos pacientes, uma vez que o isolamento social aflorou dúvidas, incertezas e dificuldades (Borges et al., 2021).

Visto que o isolamento social foi uma das recomendações para diminuir a propagação do coronavírus e a população idosa fazia parte do grupo de risco para infecção, houve a necessidade de desenvolver estratégias de cuidado para cuidar do bem-estar e da saúde mental dessa população. Alguns autores afirmam que, fatores como, medo da perda de familiares e da própria vida, problemas de saúde, dificuldade financeira, conflitos familiares, perda da independência e autonomia e o próprio isolamento, foram apontados entre os fatores que mais causaram suicídio entre os idoso durante a pandemia (Lucchini, et al., 2020).

Para minimizar os impactos decorrentes do isolamento social, foi necessária a realização de ações educativas e estímulo às atividades físicas, bem como o uso de tecnologias como o uso de celular, como também, a realização de atividades manuais, com o objetivo de apoiar a aproximação e diminuir o impacto da pandemia nessa faixa etária. Goulart, et al. (2020) apontaram os impactos da covid-19 em idosos, como precursores nas alterações da saúde mental desta população em consequência do isolamento social e a alteração nos aspectos éticos e sociais, visto que a idade muitas vezes serve como decisão de tratamento e alocação de recursos e cuidado. Desta maneira as estratégias mais adequadas é manter todos os suportes disponíveis a nível emocional, financeiro e de cuidados em saúde.

Além disso, outras medidas foram adotadas para contribuir com as intervenções de enfermagem nas ILPI e entre elas estão: a prevenção e controle para acabar com a disseminação do vírus, por meio do monitoramento da limpeza dos ambientes, como também, impedimento do contato direto entre pessoas que não estivesse fazendo uso de Equipamento de Proteção Individual (EPI) e monitoramento da entrada e saída de pessoas; o gerenciamento de cuidado, onde o foco foi diminuir o tempo em áreas comuns e replanejar as rotinas de cuidados de forma que houvesse uma diminuição nas aglomerações entre profissionais e residentes; a saúde e segurança do profissional, pois monitorava o uso de EPI entre os profissionais e residentes, além de, mapear os casos suspeitos e diagnosticados pelo covid-19, e, a comunicação com a família, que garantiu as atualizações sobre a saúde dos residentes para seus familiares (Santana et al., 2020)

O gerenciamento exercido pelo enfermeiro nas ILPI é facilitado quando as atividades estão baseadas no controle de material e treinamento de pessoal, coleta de histórico do paciente, acompanhamento das doenças já existentes e implementação do cuidado adequado. Diante desse cenário crítico e ao considerar as complexidades do paciente idoso, fica evidente que a aplicação correta do processo de enfermagem na assistência ao paciente é de fundamental importância, pois uma linguagem padronizada e um atendimento que é reavaliado constantemente contribuem na diminuição dos potenciais riscos à saúde. Por isso, a luta contra o coronavírus só provou que a equipe de enfermagem deve estar preparada e organizada para inesperados surtos, ao se basear no processo de enfermagem, o qual organiza suas ações por meio da coleta de dados, diagnóstico, resultados, intervenções e avaliação de enfermagem constantes (Santos et al., 2020).

Cardoso et al. (2020) enumeraram 11 diagnósticos de enfermagem mais encontrados em idosos que residem em ILPI e foram impactados pela pandemia, os quais foram: risco de contaminação - coletivo, risco de infecção - individual, padrão respiratório ineficaz, troca de gases prejudicada, hipertermia, diarreia, síndrome do idoso frágil, risco de lesão por pressão, integridade tissular da pele prejudicada, processos familiares interrompidos e interação social prejudicada. Os autores também 
apontaram 19 resultados de enfermagem, dentre eles, ambiente domiciliar / ILPI seguro, controle de risco comunitário: doenças contagiosas, gravidade de infecção, controle de riscos: processos infeciosos, estado respiratório: permeabilidade das vias aéreas e ansiedade.

É importante ressaltar também, que foram identificadas 33 intervenções de enfermagem que se encaixam no mesmo cenário, e dentre elas estão: controle de doenças transmissíveis - identificação de risco, controle de risco, controle de imunização/vacinação; controle do ambiente - segurança do trabalhador e controle de infecção; Supervisão - monitoração respiratória, monitoração de sinais vitais e controle de vias aéreas; controle de temperatura - administração de medicamentos, supervisão da pele, controle hídrico e eletrolítico; estimulação cognitiva e apoio ao cuidador e família; posicionamento prevenção de lesão por pressão, supervisão da pele e cuidados com lesões; promoção da resiliência e redução da ansiedade (Cardoso et al., 2020).

Assim como qualquer processo de intervenção, o processo de cuidado de enfermagem ao paciente idoso baseia-se em promoção a saúde e prevenção da doença. Foi possível observar que para implementar efetivamente qualquer tipo de assistência de enfermagem, a equipe deve estar preparada, possuir conhecimento e práticas fundamentais que garantam a efetivação do cuidado. Ao se tratar do enfermeiro como gerenciador da assistência prestada ao paciente durante a pandemia, sendo esse paciente um idoso e residente em ILPI, alguns fatores foram levados em consideração, fatores esses que incluem impactos e mudanças nas rotinas dessas instituições, do pessoal de enfermagem e dos idosos residentes.

\section{Considerações Finais}

Inúmeros são os fatores que influenciam na transferência do idoso do seu ambiente familiar para as ILPI, mas o principal deles é o objetivo da família em garantir que esse idoso seja assistido integralmente por profissionais capacitados. Porém, devido a maior parte da população idosa apresentar doenças crônicas e problemas relacionados aos sistemas motor e imunológico, as infecções podem ser facilmente desenvolvidas. Por conta disso, as ILPI demonstraram ser ambientes nos quais poderia ocorrer a disseminação do vírus do covid-19, o que de fato aconteceu em muitas instituições e resultou na contaminação de inúmeros idosos. Os estudos analisados indicaram que os pacientes apresentaram quadro clínicos diferenciados, como aparecimentos de sintomas físicos e mentais, em menor ou maior gravidade.

O enfermeiro como membro principal do sistema de gerenciamento das ILPI, contribui para coordenar a implantação de medidas de cuidado, com o propósito de promover a saúde, prevenir e controlar a disseminação do vírus. Cabe ao enfermeiro adotar estratégias para diminuir o risco de infecção e mortalidade. É evidente os enormes desafios encontrados por tais profissionais em implantar medidas de prevenção e cuidado contra uma doença pouco conhecida naquele momento e em tão pouco tempo.

Foi possível constatar que, quanto maior a capacitação e organização da assistência do pessoal de enfermagem melhores são os resultados obtidos na segurança da assistência a população idosa e que o enfermeiro precisa estar preparado para agir em diferentes situações, até mesmo em emergências. Apesar da importância de estudos na área de atenção a saúde do idoso, foram encontradas poucas publicações relacionadas especificamente a atuação da enfermagem e dessa forma, espera-se que este estudo contribua para estimular novas produções científicas a respeito deste assunto.

\section{Referências}

Alexandre, L. B. S. P \& Nunes, M. I. (2020) Problematização sobre a pandemia da COVID-19 como auxílio na formação de enfermeiras/os. Revista Nursing. 23(266). 4294-4300. http://dx.doi.org/10.36489/nursing.2020v23i266p4294-4307.

Barbosa, I. R et al. (2020) Incidência e mortalidade por COVID-19 na população idosa brasileira e sua relação com indicadores contextuais: um estudo ecológico. Revista. Brasileira de geriatria gerontologia. 23(1). http://dx.doi.org/10.1590/1981-22562020023.200171. 
Bitencourt, S. M \& Andrade, C. B. (2021). Trabalhadoras da saúde face à pandemia: por uma análise sociológica do trabalho de cuidado. Revista Ciência \& Saúde Coletiva da Associação Brasileira de Saúde Coletiva. 26(3). 1013-1022. http://dx.doi.org/10.1590/1413-81232021263.42082020.

Borges, E. M. N et al. (2021). Percepções e vivências de enfermeiros sobre o seu desempenho na pandemia da covid-19. Revista da Rede de Enfermagem do Nordeste. 22: e60790. http://dx.doi.org/10.15253/2175-6783.20212260790.

Cardoso, R. S. S et al. (2020). Diagnóstico, resultados e intervenções de enfermagem ao idoso frágil, acometido por COVID-19: uma análise documental. Research, Society and Development. 9(9): e239997109. http://dx.doi.org/10.33448/rsd-v9i9.7109.

Hammerschmidt, K. S. A \& Santana, R. F. (2020) Saúde do idoso em tempos de pandemia COVID-19. Revista Cogitare enfermagem. 25. e72849. http://dx.doi.org/10.5380/ce.v25i0.72849.

Cesário, J. M. S.; Flauzino, V. H. P \& Mejia, J. V. C. (2020) Metodologia científica: Principais tipos de pesquisas e suas caraterísticas. Revista Científica Multidisciplinar Núcleo do Conhecimento. 5(11). 23-33. http://dx.doi.org/10.32749/nucleodoconhecimento.com.br/educacao/tipos-de-pesquisas.

Gomes, L. O et al. (2020). Qualidade de vida de idosos antes e durante a pandemia da COVID-19 e expectativa na pós-pandemia. Revista Kairós: Gerontologia. 23(28). 9-28. http://dx.doi.org/10.23925/2176-901X.2020v23i0p09-28.

Goulart, L et al. (2020). A pandemia por COVID-19 e o paciente idoso: quais as necessidades de atenção em saúde para esse grupo populacional? Disciplinarum Scientia Saúde, v. 21(2). 277-286. http://dx.doi.org/10.37777/dscs.v21n2-023.

Leite, C et al. (2020). Cuidados nutricionais para idosos com doença pulmonar obstrutiva crônica em tempos de covid-19. Diálogos em Saúde. 3(1). 77-90.

Lima, L. S et al. Processo de enfermagem para pacientes com manifestações respiratórias da COVID-19. Revista de Enfermagem UFPE online. 15(1): e245345, 2021. http://dx.doi.org/10.5205/1981-8963.2021.245345

Lucchini, M. L. K et al. (2020). Fatores de risco para o suicídio em idosos antes e durante o período de confinamento por COVID-19. Research, Society and Development. 9(12): e37391211105. http://dx.doi.org/10.33448/rsd-v9i12.11105.

Marins, A. M. F et al. (2020). A saúde da pessoa idosa no contexto da pandemia pelo coronavírus: considerações para a enfermagem. Revista de Enfermagem do Centro-Oeste Mineiro. 10: e3789. http://dx.doi.org/10.19175/recom.v10i0.3789

Melo, M. A. M et al. (2020). Ações educativas aos idosos frente a pandemia de sars-cov-2: estudo de revisão. Anais do Congresso de Geriatria e Gerontologia do UNIFACIG. 1(1).

Menezes, T. M. O et al. (2020). Telemonitoramento a ILPI para idosos frente às infecções por coronavírus covid-19. Revista Brasileira de Enfermagem. 73(2). 18. http://dx.doi.org/10.1590/0034-7167-2020-0350.

Monteiro, J. K. M. F et al. (2020). Recomendações aos cuidadores e familiares de idosos mediante o COVID-19. Research, Society and Development. 9(11): e4039119798. http://dx.doi.org/10.33448/rsd-v9i11.9798.

Moraes, E. N et al. (2020). Covid-19 nas ILPI para idosos: estratégias de rastreamento laboratorial de propagação da doença. Revista Ciência \& Saúde Coletiva. 25(9). 3445-3458. http://dx.doi.org/10.1590/1413-81232020259.20382020.

Oliveira, A. S. V.; Machado, J. C \& Dadalto, L. (2020). Cuidados paliativos e autonomia de idosos expostos à covid-19. Revista Bioética. 28(4). http://dx.doi.org/10.1590/1983-80422020284422

Peters, M. D et al. (2015). Guidance for conducting systematic scoping reviews. International Journal of Evidence-Based Healthcare. 13(3). 141-146. http://dx.doi.org/10.1097/XEB.0000000000000050.

Reis, L. M et al. (2020). Atuação da enfermagem no cenário da pandemia COVID-19. Nursing (São Paulo). 23(269). 4765-4772. http://dx.doi.org/10.36489/nursing.2020v23i269p4765-4772.

Rodríguez, A. M. M. M. et al. (2020). Vacinação contra influenza no enfrentamento da COVID-19: integração ensino-serviço para formação em enfermagem e saúde. Escola Anna Nery Revista de Enfermagem. 25(spe): e20200379 DOI:10.1590/2177-9465-EAN-2020-0379.

Santana, R. F. et al (2020). Recomendações para o enfrentamento da disseminação da COVID-19 em Instituições de Longa Permanência para Idosos. Revista Brasileira de Enfermagem, 73.

Santos, G. S. N. F, et al. (2021) Análise da estrutura assistencial em saúde para com os idosos na vigência do covid-19. Anais do VII CIEH. Congresso internacional de envelhecimento humano.

Santos, J. R. L et al. (2020). Repercussões da COVID-19 para o cotidiano da pessoa idosa. Revista de Divulgação Científica Sena Aires. 9(1). 576-582. http://dx.doi.org/10.36239/revisa.v9.nesp1.p576a582.

Santos, J. M. S., Ribeiro, L. C. R. B., \& Lopes, R. F. (2020). Ações educativas em ILPI para idosos: uma revisão narrativa da literatura. Brazilian Journal of Development, 6(3), 12140-12152.

Souza, S. S et al. (2021). Influência da cobertura da atenção básica no enfrentamento da COVID-19. Journal Health Núcleo de Pesquisa e Extensão em Política, Planejamento, Organização e Práticas (individual e Coletiva) em Saúde. 6(1). 1-21. http://dx.doi.org/10.30681/252610104994.

Souza, T. A et al. (2021). Vulnerabilidade e fatores de risco associados para Covid-19 em idosos institucionalizados. Revista Eletrônica Acervo Saúde 13(2): e5947. http://dx.doi.org/10.25248/reas.e5947.2021.

Seixas, C. T et al. (2020). A crise como potência: os cuidados de proximidade e a epidemia pela Covid-19. Interface - Comunicação, Saúde, Educação. 25(1): e200379. http://dx.doi.org/10.1590/interface.200379. 